BULL. AUSTRAL. MATH. SOC.

\title{
Extensions of a semilattice by an inverse semigroup
}

\section{D.G. Green}

The structure of inverse semigroup extensions of one inverse semigroup $R$ by any other is analyzed in the case where $R$ is a semilattice. Both a representation and method of construction are given. A brief preliminary examination is made of a certain class of congruences, on inverse semigroups, which are intimately related to such extensions.

\section{Introduction}

A substantial part of group theory relates to the concept of group extensions. Since semigroup theory puts much of group theory into a more general context, it is of some interest to try to generalize this concept so as to deal with semigroup and, in particular, inverse semigroup extensions.

By an "inverse semigroup extension" we shall here understand the following: if $R, S$, and $T$ are inverse semigroups, then $S$ will be called an "(inverse semigroup) extension of $R$ by $T$ " if and only if there is at least one homomorphism $f$ (say) of $S$ onto $T$ for which $E(T) f^{-1} \cong R$ (where $E(T)$ denotes the set of idempotents of $T$ ). Occasionally we may assume, for convenience, that $R \subseteq S$.

Wuch yet remains to be done towards developing an adequate theory of inverse semigroup extensions. Coudron [3] and D'Alarcao [4] have

Received 12 February 1973. Communicated by G.B. Preston. This research was supported by a Commonwealth Postgraduate Award. The author's thanks go to Professor G.B. Preston for his help at every stage of this work. 
completely described one special case - idempotent-separating extensions wherein the $f$ above, when restricted to $E(S)$, is injective. In addition, a lot of recent work has centred around describing the structure of particular classes of inverse semigroups. In a number of cases, the inverse semigroup involved has been treated effectively as an extension of its semilattice of idempotents by some other inverse semigroup (usually a group). The characterization of free inverse semigroups, by Scheiblich [7] and others, is a notable example of this. Generalizing such characterizations, the present account describes another special case of inverse semigroup extensions - that in which $R$ in the definition is a semilattice.

In what follows, "s" denotes the natural partial order on an inverse semigroup, " $e_{x}$ " represents $x x^{-1}$ (the left identity of $x$ ), and for a congruence $\rho$, " $x \rho$ " stands for the $\rho$-class containing $x$ while " $\nu$ " denotes the natural map of $S$ onto $S / \rho$. All other definitions and notation follow the conventions of [1] and [2].

\section{Idempotent-determined congruences}

Let $\rho$ be a congruence on an inverse semigroup $S$. By its "kernel" (kero) we shall mean the union of its kernel normal system (see [2] page $60)$.

DEFINITION. A congruence $\rho$ on an inverse semigroup $S$ will be referred to as "idempotent-determined" if and only if ker $\rho=E(S)$.

Such congruences are central to our discussion. If $\rho$ is idempotentdetermined on $S$, then $v$ has the properties required by the definition to make $S$ an extension of $E(S)$ by $S / \rho$. Conversely, given an extension $S$ of a semilattice $E$ by an inverse semigroup $T$, then if $f: S \rightarrow T$ has the properties required by the definition, the congruence

$f f^{-1}$ is idempotent-determined. We start our account, therefore, by deriving some pertinent results about such congruences.

PROPOSITION 1. Let $S$ be an inverse semigroup. Every idempotentdetermined congruence on $S$ is contained in the minimum group congruence and the set of idempotent-determined congruences on $S$ forms a principal ideal in the lattice of all congmiences on $S$. 
Proof. The first assertion is immediate, as the kernel normal system of the minimum group congruence on $S$ consists of just one class, and this contains $E(S)$.

The kernel of the minimum congruence on $S$ is necessarily $E(S)$, so there is always at least one idempotent-determined congruence on $S$. Let $\tau$ denote the least congruence containing all idempotent-determined congruences (the transitive closure of their union). Suppose xte, where $x \in S$ and $e \in E(S)$. Then there are sequences $\rho_{0}, \ldots, \rho_{n}$ of idempotent-determined congruences and $x_{1}, \ldots, x_{n}$ of elements of $S$ such that $e \rho_{0} x_{1}, x_{1} \rho_{1} x_{2}, \ldots, x_{n} \rho_{n} x$. As $k e r \rho_{i}=E(S)$ for every $i \leq n$, each $x_{i}$, and by induction $x$ also, must belong to $E(S)$. Thus $\tau$ is idempotent-determined and, by its definition, must be maximum with this property. Since the set of idempotent-determined congruences on $S$ consists of just those congruences contained in $\tau$, it forms a principal ideal in the lattice of all congruences on $S$.

Notation. The maximum idempotent-determined congruence on $S$ will be denoted by $\tau(S)$,

LEMMA 1. Let $\rho$ be a congruence on an inverse semigroup $S$. Then $p$ is idempotent-determined if and only if no two distinct p-equivalent elements of $S$ have the same left identity.

Proof. Suppose that $\rho$ is idempotent-determined. From the definition of a congruence in terms of its kernel normal system ([2] page 60 ) and since $\operatorname{ker} \rho=E(S)$, we see that $\rho \subseteq\left\{(a, b): a b^{-1} \in E(S)\right\}$. Then for any $\rho$-equivalent elements $x$ and $y$, as their inverses are also $\rho$-equivalent, both $x y^{-1}$ and $x^{-1} y$ belong to $E(S)$. If further, $e_{x}=e_{y}$, it follows that $x=y y^{-1} x=y x^{-1} y$. Then, since $y x^{-1}\left(=x y^{-1}\right) \in E(S)$, we have $x \leq y$. Likewise it follows $y \leq x$, and hence $x=y$.

Conversely, if $\rho$ is not idempotent-determined, then there exist an idempotent $e$ and some $x \xi E(S)$ with $x p e$. But it follows that $e_{x} p e$, whence $x$ and $e_{x}$ are distinct $p$-equivalent elements with the same left 
identity.

The next result is essential to the constructions which follow. By an "ideal" in a semilattice $E$ we mean any subset $I$ of $E$ such that $i \in I$ and $x \leq i$ imply $x \in I$.

LEMMA 2. Let $\rho$ be an idempotent-determined congruence on an inverse semigroup $S$ and let $x$ belong to $S$. Then $e_{x}{ }^{\rho}$ is a subsemiLattice of $E(S)$ and $x \rho$ is order-isomorphic to an ideal of $e_{x} \rho$.

Proof. The first assertion follows from the fact that for any idempotents $e$ and $f, e \rho=f \rho=e_{x} \rho$ implies $e f \rho=e_{x} \rho$.

To prove the second assertion, define $E_{t}=\left\{e_{s}: s \nu=t, s \in S\right\}$ for each $t \in S / \rho$. For any $t \in S / \rho$, if there exist $x$ and $f$ in $S$ such that $e_{x} \in E_{t}$ and $f \leq e_{x}$, with $f \nu=e_{t}$, then $f=e_{f x}$ and $(f x) \nu=t$, giving $f \in E_{t}$. So for every $t \in S / \rho$, the set $E_{t}$ is an ideal in $e_{t} v^{-1}$.

Now define $\psi_{t}: E_{t} \rightarrow t \nu^{-1}$, for each $t \in S / \rho$, by $e_{x} \psi_{t}=x$ for every $e_{x} \in E_{t}$. That $\psi_{t}$ is well-defined follows from Lemma $I$ and the fact that $E_{t}$ is contained in a single congruence class. That it is oneto-one is immediate from its definition and that it is onto $t v^{-1}$ follows from the definition of $E_{t}$.

Suppose $x v=y \nu=t$ and $x \leq y$. Then $x=e_{x} y$, giving $x \psi_{t}^{-1}=e_{x}^{e} y \leq e_{y}=y \psi_{t}^{-1}$. Conversely, if $e \leq f$ in $E_{t}$, then by checking in the definition of $\psi_{t}$, we see that $e \psi_{t}=e\left(f \psi_{t}\right)$. So $e \psi_{t} \leq f \psi_{t}$. We have now established that each $\psi_{t}$ is an order-isomorphism and the second assertion follows.

\section{Representation of extensions}

In this section we shall take $S$ to be an inverse semigroup extension of a semilattice $E(=E(S))$ by an inverse semigroup $T$, with $\phi$ 
denoting a homomorphism of $S$ onto $T$ of the type required by the definition of extension. We now construct a representation of $S$.

With the subsets $E_{t}, t \in T$, of $E$ as given in the previous section define (where $T$ replaces $S / \rho$ in the obvious way)

$$
S^{\prime}=\left\{(e, t): t \in T, e \in E_{t}\right\} \text {. }
$$

Further, for each element $(e, t)$ of $S^{\prime}$, define a map $\phi(e, t): E \rightarrow E$ by

$$
f \phi(e, t)=\left(e \psi_{t}\right) f\left(e \psi_{t}\right)^{-1}
$$

for every $f \in E$ (with $\psi_{t}$ as before). Finally, define an operation $\circ$ on $S^{\prime}$ by

$$
(e, t) \circ(f, u)=(f \phi(e, t), t u) \text {, for all }(e, t),(f, u) \text { in } S^{\prime} \text {. }
$$

LEMMA 3. The maps $\phi(e, t),(e, t) \in S^{\prime}$, and $\circ$ are well-defined and each $\phi(e, t)$ is a homomorphism.

Proof. It follows from the definition that for any $f \in E, f \phi(e, t)$ is idempotent in $S$, so each $\phi(e, t)$ is well-defined.

Suppose $f \in E_{u}, u \in T$. Then for any $(e, t) \in S^{\prime}$,

$$
\begin{aligned}
& f \phi(e, t)=\left(e \psi_{t}\right)\left(f \psi_{u}\right)\left(f \psi_{u}\right)^{-1}\left(e \psi_{t}\right)^{-1} \text {. But }\left[\left(e \psi_{t}\right)\left(f \psi_{u}\right)\right] \phi=t u \text {, so } \\
& f \phi(e, t) \in E_{t u} \text { and this shows that o is well-defined. }
\end{aligned}
$$

$$
\begin{aligned}
& \text { Finally, for any }(e, t) \text { in } S^{\prime} \text {, any } f, g \text { in } E, \\
& \qquad \begin{aligned}
f g \phi(e, t) & =\left(e \psi_{t}\right) f g\left(e \psi_{t}\right)^{-1} \\
& =\left(e \psi_{t}\right) f\left(e \psi_{t}\right)^{-1}\left(e \psi_{t}\right) g\left(e \psi_{t}\right)^{-1} \\
& =f \phi(e, t) g \phi(e, t) .
\end{aligned}
\end{aligned}
$$

So $\phi(e, t)$ is a homomorphism.

The pair $\left(S^{\prime}, 0\right)$ is thus a groupoid and we can at once use it to represent $S$ in terms of $E$ and $T$.

THEOREM 1. With $S$ and $S^{\prime}$ as described above:

(i) $S$ is isomorphic to $S^{\prime}$; 
(ii) $E\left(S^{\prime}\right)=\left\{(e, f): f \in E(T)\right.$ and $\left.e \in E_{f}\right\}$.

Proof. (i) Define $\theta: S+S^{\prime}$ by $x \theta=\left(e_{x}, x \phi\right)$, for every $x \in S$. By definition of the $E_{t}$, each $e_{x}$ belongs to $E_{x \phi}$, so $\theta$ is a well-defined map. It is onto $S^{\prime}$ since for any $(e, t) \in S^{\prime}$, $\left(e \psi_{t}\right) \theta=(e, t)$. It is one-to-one since if $x \theta=y \theta$, then $\left(e_{x}, x \phi\right)=\left(e_{y}, y \phi\right)$, giving $e_{x}=e_{y}$ and $x \phi=y \phi$. Then, as $\phi \phi^{-1}$ is idempotent-determined, $x=y$. Finally, $\theta$ is a homomorphism since

$$
\begin{aligned}
x \theta \circ y \theta & =\left(e_{y} \phi\left(e_{x}, x \phi\right),(x \phi)(y \phi)\right) \\
& =\left(x y y^{-1} x^{-1},(x y) \phi\right) \\
& =(x y) \theta, \text { for any } x \text { and } y \text { in } S .
\end{aligned}
$$

(ii) For any $(e, x) \in E\left(S^{\prime}\right)$, we have $(e \phi(e, x), x x)=(e, x)$, whence $x \in E(T)$. Conversely, $f \in E(T)$ and $e \in E_{f}$ gives $e \phi(e, f)=e$ (since $e \psi_{f}$ must be idempotent), whereupon $(e, f)$ is idempotent in $S^{\prime}$.

\section{Construction of extensions}

We shall now derive a method for constructing extensions of a given semilattice by any suitable inverse semigroup. To ensure that all of the extensions possible can be produced this way, it suffices to show that the construction in the previous section can be obtained by our method. To do this we shall first obtain some properties of the above representation. These will state necessary conditions that our construction must satisfy if it is to provide extensions. It will turn out that they also give sufficient conditions .

LEMMA 4. In the representation, the sets $E_{t}, t \in T$, and mops $\phi$ and $\phi(e, t),(e, t) \in S^{\prime}$, satisfy:

(1) each $E_{t}$ is an ideal in $\left(e_{t}\right)_{\phi^{-1}}$;

(2) if $e \in E(T)$, then $E_{e}=e \phi^{-1}$;

(3) for each $t \in T$, the sets $E_{t}$ and $E_{\mathcal{u}}\left(u=t^{-1}\right)$ are 
isomorphic under a map ${ }^{*} t: E_{t} \rightarrow E_{u}$, such that

$*_{u}=\left(*_{t}\right)^{-1} \quad\left(e_{t}\right.$ will be denoted by $e^{*}$ whenever the

$t$ is understood);

(4) if $g \in E(T)$, then $e \phi(f, g)=$ ef, for each $e \in E(S)$

and every $f \in E_{g}$;

(5) for all $t \in T$ and $e \in E_{t}$,

$$
e \phi\left(e^{*}, t^{-1}\right)=e^{*},
$$

(6) for any $(e, t),(f, u)$ in $S^{\prime}$,

$$
\phi(e, t) \phi(f, u)=\phi(e \phi(f, u), u t) \text {. }
$$

Proof. (1) Proven in Lemma 2.

(2) If $e \in E(T)$, then $E_{e}=\left\{e_{x}: x \phi=e\right\}=e \phi^{-1}$, as $\phi \phi^{-1}$ is idempotent-determined.

(3) Since $\phi$ is a homomorphism, $x \mapsto x^{-1}$ is a bifection of $t \phi^{-1}$ onto $t^{-1} \phi^{-1}$; also, as $x \leq y$ if and only if $x^{-1} \leq y^{-1}$, this map is an order-isomorphism. So, from Lemma 2 , we have the order-isomorphisms

$$
E_{t} \cong t \phi^{-1} \cong t^{-1} \phi^{-1} \cong E_{u} \text {. }
$$

The resulting isomorphism is the mapping ${ }^{*}: x x^{-1} \mapsto x^{-1} x$, for $x \phi=t$, which clearly has the desired properties.

(4) If $g \in E(T)$, then necessarily $f \phi=g$ and hence $f \psi_{g}=f$, for any $f \in E_{g}$. So $e \phi(f, g)=f e f=e f$, for all $e \in E$.

(5) By definition,

$$
\begin{aligned}
e \phi\left(e^{*}, t^{-1}\right) & =\left(e^{*} \psi_{u}\right) e\left(e^{*} \psi_{u}\right)^{-1},\left(u=t^{-1}\right) \\
& =\left(e \psi_{t}\right)^{-1} e\left(e \psi_{t}\right), \text { by the proof of }(3), \\
& =e^{*} .
\end{aligned}
$$

(6) For any $g \in E$, both sides of the equation give $(x y) g(x y)^{-1}$, 
where $x=f \psi_{u}$ and $y=e \psi_{t}$.

REMARK. Property ( 1 ) is in fact implied by the right side of the equation in (6), which requires that $e \phi(f, u) \in E_{u t}$. However, we retain (I) as a condition, for completeness.

The definition of inverse semigroup extensions puts restrictions upon the class of inverse semigroups by which we can extend a given inverse semigroup. In accordance with this, let $E$ be a semilattice and $T$ be any inverse semigroup such that there is a homomorphism $\phi: E \rightarrow T$ which is onto $E(T)$. Now, treating the results in Lemma 4 as conditions, we can form a set $S^{\prime}$ just as before, by selecting for the sets $E_{t}$ any subsets of $E$ satisfying conditions (1) to (3). If, further, we can choose, for each $(e, t) \in S^{\prime}$, endomorphisms $\phi(e, t): E \rightarrow E$ satisfying conditions (4) to (6), then an operation $\circ$ can be defined on $S$ ' exactly as before. The following properties now characterize this construction:

THEOREM 2. With $E, T, \phi$ and $S^{\prime}$ as above:

(a) $\left(S^{\prime}, 0\right)$ is an inverse semigroup;

(b) $E\left(S^{\prime}\right)=\{(e, e \phi): e \in E\}$;

(c) $T$ is a homomorphic image of $S^{\prime}$ under

$$
\alpha:(e, x) \mapsto x ;
$$

(a) S' is an inverse semigroup extension of $E$ by $T$;

(e) the maximum idempotent-determined congmences on $S^{\prime}$ and $T$ are related by

$$
\tau\left(S^{\prime}\right)=\alpha \tau(T) \alpha^{-1} .
$$

Proof. (a) Condition (6) implies that $E_{u} \phi(e, t) \subseteq E_{t u}$, for every $u \in T$ and $(e, t) \in S^{\prime}$, hence ensuring that $\circ$ is well-defined. The associativity of $\circ$ also follows from condition $(6)$ by application in the definition of 0 . Conditions (3) and (5) ensure that $\left(e^{*}, t^{-1}\right)$ will be an inverse of $(e, t)$, for any $(e, t) \in S^{\prime}$.

From the definition of 0 it immediately follows that if $(e, x)$ and $(f, y)$ say, are idempotent in $S^{\prime}$, then $x$ and $y$ must be idempotent in 
$T$. But then, from condition (4), it follows that

$$
(e, x) \circ(f, y)=(e f, x y)=(f e, y x)=(f, y) \circ(e, x) .
$$

So idempotents commute in $S^{\prime}$ and this completes the proof that $S^{\prime}$ is an inverse semigroup.

(b) Condition (2) ensures that each $(e, e \phi)$ beiongs to $S^{\prime}$. That it is idempotent follows from condition (4) and the fact that $\phi$ is a homomorphism.

Conversely, we saw above that $(e, x) \in E\left(S^{\prime}\right)$ implies that $x$ is idempotent in $T$. Since $e \in e_{x} \phi^{-1}$, it follows that $e \phi=x$. Note that this result makes $E\left(S^{\prime}\right)$ isomorphic to $E$ under $(e, e \phi) \mapsto e$.

(c) Since, under multiplication in $S^{\prime}$, the second components of elements of 'S' combine by multiplication in $T, \alpha$ is immediately a homomorphism. That it is onto $T$ follows from the construction of $S^{\prime}$.

(d) The kernel of $\alpha \alpha^{-1}$ is $E\left(S^{\prime}\right)$ which, from (b) above, is isomorphic to $E$. So $E(T) \alpha^{-1} \cong E$ and this, together with (c), gives the result.

(e) For any elements $(e, t)$ and $(f, u)$ of $S^{\prime}$, $(e, t)\left(\alpha \tau(T) \alpha^{-I}\right)(f, u)$ if and only if $t \tau(T) u$. Thus, since $\tau(T)$ is idempotent-determined, and $(e, f)$ is idempotent in $S^{\prime}$ if and only if $f$ is in $T$, the congruence $\alpha \tau(T) \alpha^{-1}$ is idempotent-determined also.

Given $\tau\left(S^{\prime}\right)$ we can define a congruence $\tau$ on $T$ by $s \tau t$ if and only if there exist $(e, s)$ and $(f, t)$ in $S^{\prime}$ with $(e, s) \tau\left(S^{\prime}\right)(f, t)$. As $\operatorname{ker\tau }\left(S^{\prime}\right)=\{(e, e \phi): e \in E\}$ it follows that $\operatorname{ker} \tau=E(T)$. So $\tau \subseteq \tau(T)$. Then $(e, s) \tau\left(s^{\prime}\right)(f, t)$ implies $(e, s) \alpha \tau(f, t) \alpha$, whence $(e, s) \alpha \tau(T)(f, t) \alpha$. This gives $\tau\left(S^{\prime}\right) \subseteq \alpha \tau(T) \alpha^{-1}$ and the result follows.

\section{Comments and examples}

(1) In our construction of extensions, if both $E$ and $T$ are semilattices, then $\phi$ must be onto $T$ and it follows, from result $(b)$ of Theorem 2, that $S^{\prime}$ is isomorphic to $E$.

(2) In the construction, if $\phi$ is an embedding of $E$ into $T$, then 
$E$ must be isomorphic to $E(T)$, making $S^{\prime}$ isomorphic to $T$.

(3) If $T$ is a group, then the inverse semigroup $E \times T$, with usual product, is an extension of $E$ by $T$. This follows via the construction of Theorem 2, taking $E_{t}=E$, and mappings $f \phi(e, t)=e f$ and ${ }^{*} t={ }^{1} E$, for each $t \in T$ and $e \in E_{t}$.

(4) Any F-inverse semigroup (see [5]) is an extension of its semilattice of idempotents by its maximum group homomorphic image. To show this, a construction for F-inverse semigroups can be obtained by specifying extra conditions in our construction above. The following extra conditions suffice to meet the requirements given by McFadden and $0^{\prime} C a r r o l l$ in [5]:

(a) $T$ is a group;

(b) each $E_{t}$ is a principal ideal of $E$;

(c) for every $(e, t) \in S^{\prime}, \phi(e, t)=\phi\left(I_{t}, t\right)$ (called $\phi_{t}$ in [5]), where ${ }^{1} t$ is maximum in $E_{t}$;

(d) $1 \phi\left(1_{t}, t\right)=I_{t}$, where 1 is maximum in $E$.

(5) The free inverse semigroup $I_{X}$ on a set $X$ is an extension of its semilattice of idempotents by $G_{X}$ - the free group on $X$. This is shown by checking that the representation of $I_{X}$ (as given in [6] say, which is readily adaptable to our notation) satisfies the conditions we have given.

\section{References}

[1] A.H. Clifford and G.B. Preston, The algebraic theory of semigroups, Volume I (Math. Surveys 7 (I), Amer. Math. Soc., Providence, Rhode Island, 1961).

[2] A.H. Clifford and G.B. Preston, The algebraic theory of semigroups, Volume II (Math. Surveys 7 (II), Amer. Math. Soc., Providence, Rhode Island, 1967). 
[3] A. Coudron, "Sur les extensions de demi-groupes réciproques", BulZ. Soc. Roy. Sci. Liège 37 (1968), 409-419.

[4] H. D'Alarcao, "Idempotent-separating extensions of inverse semigroups", J. Austral. Math. Soc. 9 (1969), 211-217.

[5] R. McFadden and L. O'Carroll, "F-inverse semigroups", Proc. London Math. Soc. (3) 22 (1971), 652-666.

[6] G.B. Preston, "Free inverse semigroups", J.Austral. Math. Soc. (to appear).

[7] H.E. Scheiblich, "Free inverse semigroups", Semigroup Forum 4 (1972), 351-359.

Department of Mathematics,

Monash university,

Clayton,

victoria. 